\title{
X-ray observations of IC 348 in light of an updated cluster census
}

\author{
B. Stelzer, G. Micela, E. Flaccomio and S. Sciortino \\ INAF - Osservatorio Astronomico di Palermo, Piazza del Parlamento 1, I-90134 Palermo, Italy
}

\begin{abstract}
IC 348 is an excellent laboratory for studies of low-mass star formation being nearby, compact and rich. A Chandra observation was carried out early in the satellite's lifetime. The extensive new data in optical and infrared wavelengths accumulated in subsequent years have changed the cluster census calling for a re-analysis of the X-ray data.
\end{abstract}

Keywords: X-rays: stars, Stars: formation, pre-main sequence, activity, coronae

PACS: 95.85.Nv, 97.10.Bt, 97.21.+a, 97.10.Ex, 97.10.Jb

\section{THE CURRENT IC 348 CLUSTER CENSUS}

IC 348 is the nearest $(\sim 310 \mathrm{pc})$, rich and compact $\left(d \sim 20^{\prime}\right)$ cluster of low-mass star formation. To date, more than 300 members in a wide mass range $\left(\sim 0.02-5 M_{\odot}\right)$ have been spectroscopically confirmed, most of them within the central cluster area of $16^{\prime} \times 14^{\prime}$. The extinction is relatively low $\left(A_{\mathrm{V}}<4\right.$ mag for most known members) and the mean cluster age is $2 \mathrm{Myr}$.

With these characteristics IC 348 is a prime laboratory for studies of low-mass star formation. A $53 \mathrm{ksec}$ of IC 348 was presented by [1, 2]. Subsequently, extensive work was done on the cluster at optical and infrared wavelengths:

- $\sim 130$ new cluster members were discovered by signatures of youth in optical lowresolution spectra and their position in the HR diagram above the $10 \mathrm{Myr}$ isochrone [3];

- the stellar parameters have been revised shifting some objects to earlier spectral type, i.e. higher mass [3];

- Young Stellar Objects (YSOs) have been classified using Spitzer photometry [4];

- rotation periods were measured for $>100$ cluster members [e.g. 5].

All these stellar properties impact on the interpretation of the X-ray data, such as the relation between X-ray luminosity and stellar parameters $\left(L_{\mathrm{bol}}, T_{\mathrm{eff}}, M\right), \mathrm{X}$-ray luminosity functions, the rotation-activity relation, etc. Therefore, we present a reanalysis of the Chandra observation of IC 348 incorporating the updated optical and infrared database. Two representations of the Chandra image are shown in Fig. 1 , 

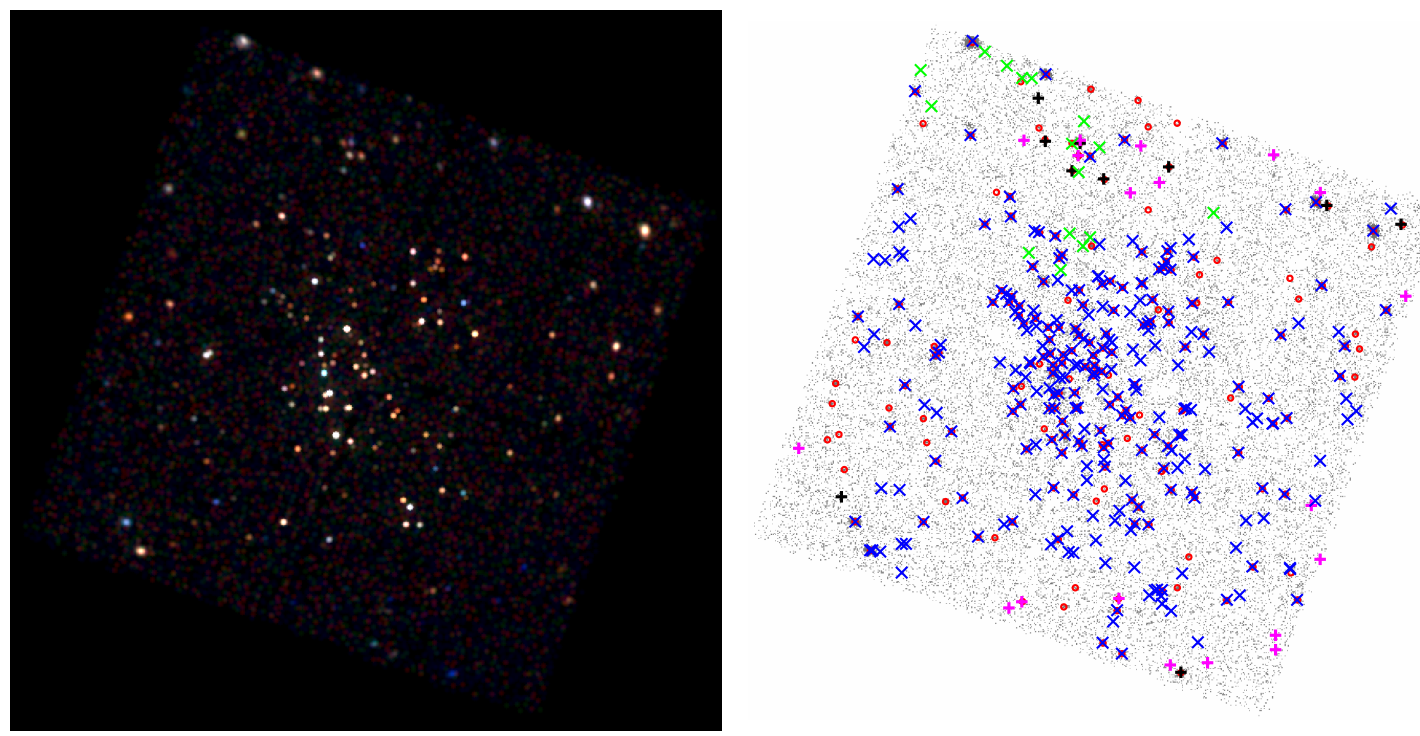

FIGURE 1. Chandra/ACIS-I image of IC 348: (LEFT) - false-color image; (RIGHT) - image with Xray sources from [1, 2] (red circles) and cluster members from the literature: blue [3], green [6], pink and black [7]; blue, green and pink sources are spectroscopically confirmed IC 348 members.

\section{DATA ANALYSIS}

In this paper we use the results from our new analysis of the X-ray data only for known IC 348 members absent from the list of detections in [1, 2]. For all detections in common with our new analysis the X-ray data from [1, 2] is used. The X-ray source list was crosscorrelated with the IC 348 master catalog compiled from the references given in Sect. 1. The re-analysis is also used to extract upper limits for undetected IC 348 members. This is mandatory for a complete X-ray census, because upper limits are not available from [1, 2] for the IC 348 members discovered after 2001.

\section{RESULTS}

\section{X-rays and evolutionary stage}

IC 348 is rich in Young Stellar Objects (YSOs) of all evolutionary stages. Class I protostars, disk-bearing Class II T Tauri stars and disk-less Class III T Tauri stars can be distinguished by the slope of their spectral energy distribution (SED) in the mid-IR. The spectral index $\alpha_{\text {SED }}$ derived from Spitzer mid-IR photometry for IC 348 members was presented by [4]. We assign the YSO class to each X-ray source using the boundaries for $\alpha_{\mathrm{SED}}$ defined by [4] and [7].

The X-ray detection statistics for the different YSO classes in IC 348 can be summarized as follows: $N_{\mathrm{det}} / N_{\mathrm{ul}}=5 / 7$ (Class I), $45 / 55$ (Class II), 36/31 (Class II/III), and $93 / 22$ (Class III) where $N_{\text {det }}$ is the number of detections and $N_{\mathrm{ul}}$ the number of undetected stars. 

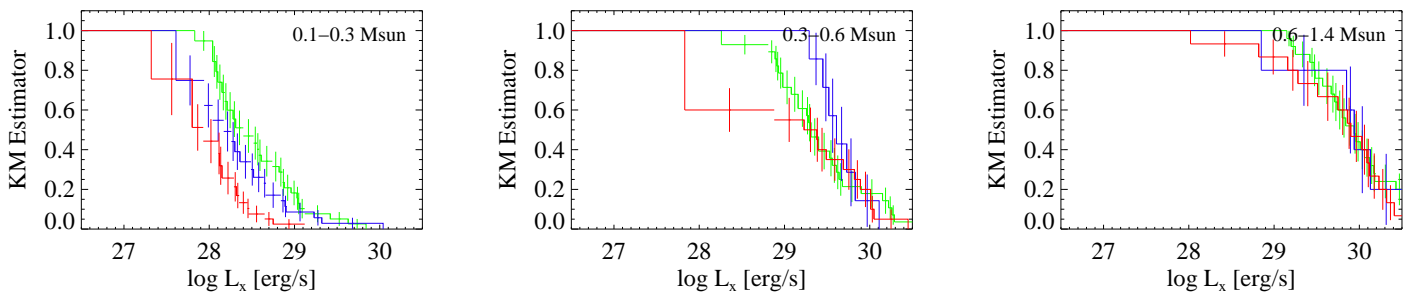

FIGURE 2. X-ray luminosity functions for the Spitzer YSO classes in different mass bins. red - Class II, blue - Class II/III, green - Class III.

\section{X-ray luminosity functions}

We computed X-ray luminosity functions (XLF) for different YSO classes (to examine the influence of disks) and for different levels of $\mathrm{H} \alpha$ emission (to examine the influence of accretion). To avoid biases related to the known dependence of $L_{\mathrm{x}}$ on stellar mass, the analysis was carried out in three mass bins. The XLF take into account the upper limits for undetected stars.

In Fig. 2] we compare the XLF of Class II, II/III, and III sources. Class I objects are not considered because of poor statistics. There is a pronounced difference in the X-ray luminosities of the three YSO classes for the lowest mass bin $\left(0.1-0.3 M_{\odot}\right)$, while for higher mass stars the XLF of the three types of YSOs can not be distinguished.

\section{X-rays and stellar parameters}

Fig. 3 shows the HR diagram and the $L_{\mathrm{x}}$ vs. $L_{\mathrm{bol}}$ diagram for IC 348 . Evidently most undetected cluster members are very-low mass stars and brown dwarfs. The sample of known IC 348 members extends to lower bolometric luminosities and masses than that of Orion, possibly due to both environmental and observational effects (e.g. higher extinction in Orion). Therefore, IC 348 is excellent for studying the relation between $\mathrm{X}$-ray activity an stellar parameters in very-low mass stars and brown dwarfs. Contrary to what was observed for Orion and Taurus [see 8, 9], we find that the relation between $L_{\mathrm{x}}$ and $L_{\mathrm{bol}}$ in IC 348 seems to be steeper than expected for constant $L_{\mathrm{x}} / L_{\mathrm{bol}}$ ratio (see Fig. 3 right). This is due to a large number of X-ray faint (undetected) low-mass stars. Note, that the fitted curve for IC 348 includes only stars with $>0.1 M_{\odot}$ where upper limits are well constrained.

Clearly, at the low-luminosity end the X-ray data are dominated by insufficiently constrained upper limits. Some hypotheses for a possible decrease of $L_{\mathrm{x}} / L_{\mathrm{bol}}$ level towards lower masses are: (i) transition from a solar-like to a convective dynamo, combined with a lower efficieny of the latter one resulting in decreased X-ray production; (ii) smaller co-rotation radius for lower-mass stars and the ensuing centrifugal disruption of the corona [12]; (iii) a mass dependent fraction of stars with X-ray emission suppressed by accretion [13]. A deeper X-ray observation is necessary to unveil the behavior of the $L_{\mathrm{x}} / L_{\mathrm{bol}}$ level for the faint and cool objects with the lowest stellar masses $\left(0.1 \ldots 0.3 M_{\odot}\right)$. 

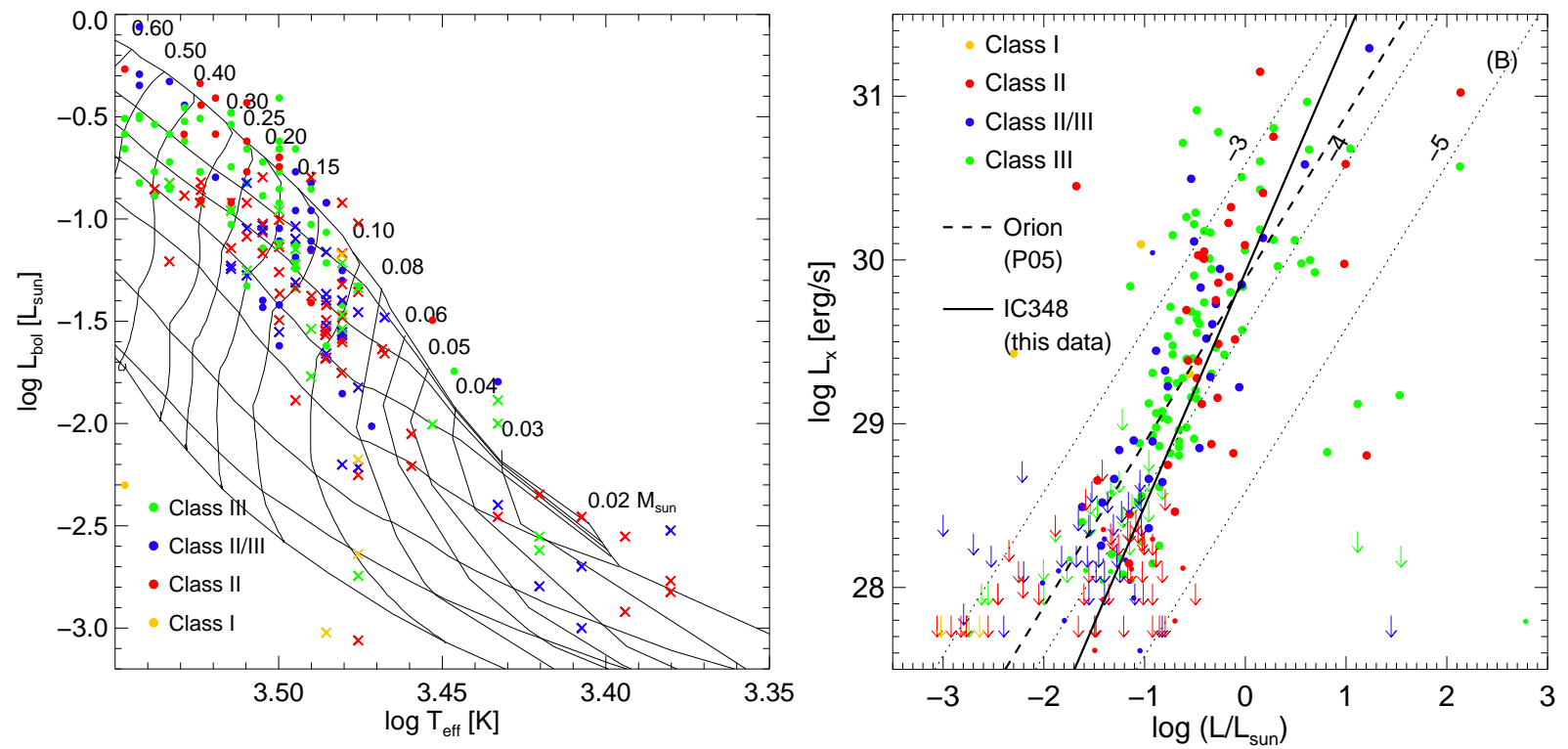

FIGURE 3.

(LEFT) - Low-mass region of the HR diagram for IC 348 compared to evolutionary tracks of [10] and [11]. filled circles - X-ray detections, crosses - non-detections;

(RIGHT) - X-ray versus bolometric luminosity; dotted lines represent constant $L_{\mathrm{X}} / L_{\mathrm{bol}}$ ratios $10^{-3}$, $10^{-4}$ and $10^{-5}$; the dashed line is the linear regression fit derived by [8] for the Orion Nebula Cluster corresponding to $\log \left(L_{\mathrm{x}} / L_{\mathrm{bol}}\right)=-3.7$; the solid line is a linear regression fit to the IC 348 members with $M \leq 1.2 M_{\odot}$ excluding some flaring objects.

\section{REFERENCES}

1. T. Preibisch, and H. Zinnecker 122, 866-875 (2001).

2. T. Preibisch, and H. Zinnecker, AJ 123, 1613-1628 (2002).

3. K. L. Luhman, J. R. Stauffer, A. A. Muench, G. H. Rieke, E. A. Lada, J. Bouvier, and C. J. Lada, ApJ 593, 1093-1115 (2003).

4. C. J. Lada, A. A. Muench, K. L. Luhman, L. Allen, L. Hartmann, T. Megeath, P. Myers, G. Fazio, K. Wood, J. Muzerolle, G. Rieke, N. Siegler, and E. Young, AJ 131, 1574-1607 (2006).

5. L. Cieza, and N. Baliber, ApJ 649, 862-878 (2006).

6. K. L. Luhman, E. A. Lada, A. A. Muench, and R. J. Elston, ApJ 618, 810-816 (2005).

7. A. A. Muench, C. J. Lada, K. L. Luhman, J. Muzerolle, and E. Young, AJ 134, 411-444 (2007).

8. T. Preibisch, Y.-C. Kim, F. Favata, E. D. Feigelson, E. Flaccomio, K. Getman, G. Micela, S. Sciortino, K. Stassun, B. Stelzer, and H. Zinnecker, ApJS 160, 401-422 (2005).

9. N. Grosso, K. R. Briggs, M. Güdel, S. Guieu, E. Franciosini, F. Palla, C. Dougados, J.-L. Monin, F. Ménard, J. Bouvier, M. Audard, and A. Telleschi, A\&A 468, 391-403 (2007).

10. I. Baraffe, G. Chabrier, F. Allard, and P. H. Hauschildt, $A \& A$ 337, 403-412 (1998).

11. G. Chabrier, I. Baraffe, F. Allard, and P. Hauschildt, ApJ 542, 464-472 (2000).

12. M. Jardine, A. C. Cameron, J.-F. Donati, S. G. Gregory, and K. Wood, MNRAS 367, 917-927 (2006).

13. E. Flaccomio, G. Micela, and S. Sciortino, A\&A 402, 277-292 (2003). 